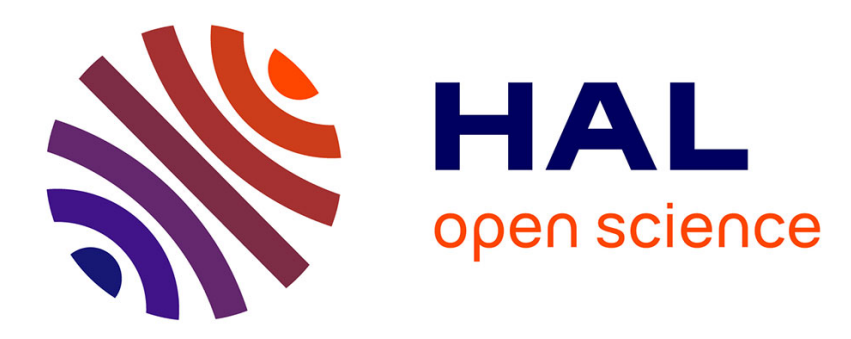

\title{
Une odeur déconcertante chez tante Léonie.
} Joël Candau

\section{To cite this version:}

Joël Candau. Une odeur déconcertante chez tante Léonie.. Sensations proustiennes, 2016. halshs01736668

\section{HAL Id: halshs-01736668 \\ https://shs.hal.science/halshs-01736668}

Submitted on 20 Mar 2018

HAL is a multi-disciplinary open access archive for the deposit and dissemination of scientific research documents, whether they are published or not. The documents may come from teaching and research institutions in France or abroad, or from public or private research centers.
L'archive ouverte pluridisciplinaire HAL, est destinée au dépôt et à la diffusion de documents scientifiques de niveau recherche, publiés ou non, émanant des établissements d'enseignement et de recherche français ou étrangers, des laboratoires publics ou privés. 
Publié : Candau Joël. 2016. Une odeur déconcertante chez tante Léonie, numéro "Sensations proustiennes" de la Revue Marcel Proust Aujourd'hui, n 13, p. 142157.

\author{
UNE ODEUR DÉCONCERTANTE CHEZ TANTE LÉONIE
}

\author{
Joël Candau (joelcandau@gmail.com) \\ Université de Nice Sophia Antipolis, LAPCOS (EA 7278)
}

\begin{abstract}
Résumé : Lors de mes visites à Combray, se souvient le narrateur, « je revenais toujours avec une convoitise inavouée m'engluer dans l'odeur médiane, poisseuse, fade, indigeste et fruitée du couvre-lit à fleurs » de tante Léonie. Que peut bien être une odeur «médiane»? Je propose ici plusieurs interprétations, littéraires, sociologiques et anthropologiques de cette odeur déconcertante, après avoir commenté quelques propriétés de la mémoire des odeurs mises en lumière par le texte proustien. L'hypothèse à mes yeux la plus plausible est que dans ce domaine si particulier des expériences olfactives, l'impropriété apparente de l'épithète "médiane " participe d'un processus de sédimentation sémantique visant à susciter chez nous, lecteurs, de multiples inférences susceptibles de satisfaire une quête du partage qui nous anime en permanence tout autant qu'elle anima l'auteur de la Recherche.
\end{abstract}

Feu d'artifice odorant dans la Recherche, l'évocation de la chambre de tante Léonie offre une véritable énigme ${ }^{1}$. Lors de mes visites à Combray, se souvient le narrateur, « je revenais toujours avec une convoitise inavouée m'engluer dans l'odeur médiane, poisseuse, fade, indigeste et fruitée du couvre-lit à fleurs » de tante Léonie (Proust 1987a : 61). Que peut bien être une odeur " médiane»? $\mathrm{Au}$ risque de quelques errements anthropologico-littéraires, je propose ici plusieurs interprétations de cette odeur étrange, après avoir commenté quelques propriétés de la mémoire des odeurs mises en lumière par le texte proustien.

\title{
1. LA MÉMOIRE OLFACTIVE ET SON LANGAGE
}

À des années de distance de la sensation originelle, la mémoire olfactive du narrateur paraît étonnamment fidèle, si l'on en juge d'après le nombre et la densité sémantique des descripteurs de l'odeur du couvre-lit. Bien connue (Candau 2001), cette ténacité des souvenirs olfactifs est particulièrement forte quand ils se rapportent à la saison de la petite enfance, notamment entre 6 et 10 ans (Chu \& Downes 2000), tranche d'âge où Proust passa ses vacances à Illiers-Combray chez sa tante paternelle. J'en ai fait le constat dans mes propres enquêtes ethnographiques (Candau 2000). Chez de nombreux chefs cuisiniers, le souvenir sensoriel des recettes familiales sature leur mémoire culinaire. Chez les parfumeurs, tel composant évoque l'odeur de la cuisine maternelle, tel autre l'odeur d'un livre lu enfant ou adolescent. Le « vécu olfactif» des œnologues structure tout aussi fermement leur perception. Par exemple, lors d'une dégustation d'un vin varois, l'un d'entre eux associe l'odeur de pêche au lointain souvenir du verger familial. Un autre rapproche l'odeur du musc de celle du vieux linge de maison que, tout enfant, il découvrit dans une malle entreposée dans le grenier de ses grandsparents.

La tonalité hédonique de la mémoire olfactive, manifeste dans le souvenir d'une "convoitise inavouée », est elle aussi amplement documentée. "Quand c'est la mémoire qui respire », observait Bachelard, «toutes les odeurs sont bonnes. » (Bachelard 1960 \& 1993 : 119). D'une enquête que j'ai menée il y a quelques années auprès de plus de 500 étudiants de l'Université de Nice Sophia Antipolis, il ressort des souvenirs olfactifs majoritairement plaisants, tant du point de vue du contenu mémorisé que du contexte qui lui est lié. Très souvent rattachés à l'enfance, ils renvoient aux vacances, aux voyages, à la nature (mer, montagne, campagne) et à la famille (odeurs et parfums des parents et grands-parents, repas dominicaux, maison familiale). 
Enfin, la perception du caractère « invasif» des odeurs, sur le mode de l'engluement ressenti par le narrateur, est attestée lors de nombreuses expériences olfactives. Les difficultés rencontrées pour se protéger des stimuli olfactifs, l'accès immédiat des messages au cerveau, tout concourt à faire de l'odorat un sens de l'intrusion dont nous sommes contraints de " partager l'apport » (Kant 1986 : 976), en particulier en présence des odeurs humaines (Baccino et al. 2010) très souvent situées sur le versant négatif de l'espace hédonique. Dans les professions qui y sont exposées, certains effluves donnent à mes interlocuteurs l'impression d'une imprégnation physique et, même, d'une pénétration. L'odeur d'un cadavre en état de putréfaction, disent les fossoyeurs, "accroche ", "pique " et "rentre dans les cheveux ». Selon un thanatopracteur, cette odeur "reste collée dans la bouche », on la " garde sur soi » pour la "porter » ensuite, tel un fardeau. En milieu hospitalier, les odeurs du corps humain évoquent également l'agression. Une odeur d'infection, estime une infirmière, "imprègne" ses vêtements et son corps. Une autre a l'impression " d'avaler les parcelles infimes du corps qui filtrent de certaines escarres ou de nécroses ». L'odeur du noyé, déclare un médecin légiste, "lourde », " collante » et qui "vient nous tartiner » est "à ne pas regarder " (Candau \& Jeanjean 2006). Ces descripteurs olfactifs font tous songer aux représentations médicales qui, aux XVIe et XVIIe siècles, conféraient aux odeurs la faculté de pénétrer l'intimité des corps (Vigarello 1985 : 18).

J'en viens maintenant à l'objet principal de ma discussion, la singularité du langage des odeurs, patente dans le texte de Proust et dans son usage du terme " médiane». Ces odeurs, comment les pensons-nous? Comme toutes les qualités sensibles, à l'aide d'«idées confuses et obscures », répondent les auteurs de La logique de Port-Royal (Arnauld \& Nicole 1992 : 64). Ces idées sont-elles toujours associées à des signes? Non, car la conceptualisation n'implique pas nécessairement une verbalisation (Pinker 1999). On peut percevoir un stimulus (sonore, visuel, olfactif, gustatif, tactile, proprioceptif) sans disposer d'un terme pour le nommer, fait relevé par Cabanis, fortement influencé par le sensualisme condillacien. "Les matériaux des idées, écrit-il dans Rapports du physique et du moral de l'homme, existent bien certainement [...] avant les signes » Cependant, ajoute-t-il, « on ne distingue les sensations qu'en leur attachant des signes qui les représentent et les caractérisent ; on ne les compare qu'en représentant et caractérisant également par des signes ou leurs rapports ou leurs différences. » Ces signes - c'est-à-dire, selon Cabanis, une langue - permettent de fixer nos propres sensations.

« Ils les retracent, et par conséquent ils les rappellent; c'est là-dessus qu'est fondé l'artifice de la mémoire. [...] Les signes rappellent donc les sensations ; ils nous font sentir de nouveau. Il en est qui restent, pour ainsi dire, cachés dans l'intérieur ; ils sont pour l'individu lui seul. Il en est qui se manifestent au-dehors ; ils lui servent à communiquer avec autrui » (Cabanis 1980 : 95 et 96).

Ce sont bien ceux-là qui intéressent l'anthropologue, si l'on admet que cette communication est la condition du partage des significations. Mais ces signes, qui permettent selon Lévi-Strauss de transcender l'opposition du sensible et de l'intelligible, rendent-ils véritablement «les qualités secondes au commerce de la vérité » (Lévi-Strauss $1964: 22)$ ?

Quand on considère l'encodage sensoriel des couleurs, cela semble vrai. Si je dis que les rideaux de mon bureau sont rouges, toute personne voyante peut se faire une idée assez précise de l'impression lumineuse qu'ils donnent, avec des variations individuelles négligeables (excepté les cas pathologiques) qui n'altèrent probablement pas la communication intersubjective. Mais lors d'une expérience olfactive, cette description de la perception à des fins de communication ne va pas de soi, pour au moins trois raisons. En premier lieu, de nombreuses odeurs restent à un niveau infraverbal. Notre exploration olfactive du monde se fait massivement de manière holistique, l'odeur étant appréhendée comme un tout sans qu'il y ait nécessairement codage verbal. Ensuite, si nous sommes plutôt habiles pour détecter les substances odorantes (parmi les composés volatils soufrés, par exemple, nous sommes capables de percevoir le furfurylthiol, un composant de l'arôme du café, au seuil extrêment faible de 5 ng.L (Filippi, Fernandez \& Duñach 2007)) et modestement compétents pour les discriminer et les catégoriser (Candau \& Wathelet 2011), aptitudes indubitablement utiles à la survie de notre espèce, nous sommes bien moins performants lorsqu'il s'agit de les décrire, même si cela n'est pas vrai de toutes les langues (Majid \& Burenhult 2014). Contrairement à «la belle systématicité du lexique des couleurs » (Boisson 1997 : 31), le lexique olfactif est souvent imprécis et instable (le même descripteur peut avoir plusieurs référents et, à un même stimulus, peuvent être 
associés plusieurs descripteurs). Si j'écris que, tout à l'heure dans la rue, j'ai senti une odeur épaisse, quelle signification les lecteurs vont-ils associer à ce descripteur? Sa compréhension, on le devine, sera moins facilement partagée que lorsque j'évoquais la couleur rouge des rideaux de mon bureau. En définitive, le langage naturel des odeurs se caractérise par une grande variation interindividuelle, ellemême fonction de la diversité de nos expériences. Les catégories olfactives peuvent être relativement précises pour un individu, mais perdre cette précision quand elles sont partagées, leurs limites dépendant étroitement d'un contexte approprié par un sujet singulier. Il en résulte que la dénomination des odeurs sous une seule étiquette est presque toujours impossible. L'objectivité de l'odeur « n'a pas été construite, dans notre culture tout au moins, par la négociation d'un partage du sens dans l'interaction verbale » (Dubois \& Rouby $1997: 16$ ).

Cette particularité de l'encodage olfactif a conduit certains chercheurs à qualifier l'odorat de sens « muet », abusivement à mes yeux. En effet, même si le langage des odeurs reste approximatif, il n'est pas arbitraire ${ }^{2}$ et il lui arrive d'être riche, voire exubérant. Beaucoup de mots (substantifs ou épithètes) sont métaphoriques, permettant ainsi de structurer partiellement l'expérience olfactive dans les termes d'une autre (vision, toucher, etc.). Cela tient notamment au fait que la mémoire olfactive enregistre en même temps que le stimulus tout son contexte sensoriel et émotionnel, phénomène connu sous le nom de syndrome de Proust. Plusieurs régions cérébrales qui participent au traitement du message olfactif marquent l'odeur d'une valeur affective (Candau 2010a), réunissent diverses informations sensorielles et jouent ainsi un rôle central pour la mise en mémoire de souvenirs qui ne sont jamais purement olfactifs, ce que suggère fort bien leur expression en langue (Candau 2010b). Là où la langue cherche à désigner certaines qualités olfactives, soutient Cassirer (1972:150-151), elle se voit contrainte à emprunter le détour de mots " tirés d'autres données de l'intuition sensible ». Chez la plupart de mes informateurs, effectivement, la description olfactive emprunte aux autres sens. Les sommeliers, par exemple, font intervenir la vision (la robe), le toucher (le volume en bouche, "gras, riche et opulent » ou bien "fluide et mince ») et bien sûr le goût proprement dit. La description multisensorielle peut être subtile : on évoque des " notes » de miel ou de résine, des «touches» de fleur ou de champignon, une "pointe» de truffe, etc. Ce langage des correspondances, on le sait, est également attesté chez les parfumeurs qui se présentent souvent comme des compositeurs jouant de notes olfactives. Le parfum Nahema, affirme son créateur Jean-Paul Guerlain, a été construit comme un morceau de musique, en cherchant à recréer le rythme lancinant du Boléro de Ravel. Le même langage multisensoriel est utilisé par les cuisiniers qui, à l'instar des parfumeurs, évoquent les "notes » aromatiques. L'un d'entre eux, par exemple, qui se considère comme un "chef d'orchestre ", assimile les odeurs à des "notes de musique ». Dans un tout autre registre, un employé des pompes funèbres décrit l'odeur d'un noyé comme " sourde, basse » alors que celle d'une personne brûlée est plus " haute ». Enfin, à propos de "l'odeur de la souffrance » dégagée selon lui par des défunts à l'agonie difficile, il évoque "le bruit de l'odeur».

De l'ensemble de ces données se dégage l'interprétation suivante : le terme "médiane », un hapax ${ }^{3}$ dans la Recherche, n'est finalement qu'une illustration du caractère multisensoriel du langage naturel des odeurs. Peut-on aller plus loin ? Je m'y hasarde, dans les hypothèses et excursus littéraires qui suivent.

\section{PARTAGER UNE ODEUR MÉDIANE?}

\section{Hypothèses littéraires}

N'étant ni proustien ni spécialiste en littérature, c'est de manière cursive et avec la plus grande humilité que j'envisage ici cinq hypothèses littéraires, ce qui est à coup sûr réducteur d'une exégèse de l'œuvre potentiellement infinie. La première d'entre elles est donnée par le contexte, au sens premier du mot. Dans l'introduction, je n'ai cité qu'un court extrait du texte où le narrateur, avant d'évoquer l'odeur médiane du couvre-lit, décrit la chambre de tante Léonie et le feu qui y brûlait entre deux briques. Celui-ci, raconte-t-il, cuisait

« comme une pâte les appétissantes odeurs dont l'air de la chambre était tout grumeleux [...], il les feuilletait, les dorait, les godait, les boursouflait, en faisant un invisible et palpable gâteau provincial, un immense " chausson » où, à peine goûtés les arômes plus croustillants, plus fins, plus réputés, mais plus secs aussi du placard, de la commode, du papier à ramages, je revenais 
toujours avec une convoitise inavouée m'engluer dans l'odeur médiane, poisseuse, fade, indigeste et fruitée du couvre-lit à fleurs » (Proust 1987a : 61).

Selon Geneviève Idt, l'épithète «médiane » signifie ceci : «qui est situé au milieu, comme la compote de pommes au milieu du chausson, et le « lit de milieu » au centre de la pièce » ${ }^{4}$ (Idt 1975). Une série d'analogies spatiales se répondraient donc l'une l'autre dans le récit du narrateur. En cela, l'acception du terme "médiane » correspondrait à celle communément observée dans plusieurs textes littéraires contemporains de Proust (Base Frantext).

Par bien des côtés, la Recherche est « une enquête sociale » (Brunet 1983 : 149). L'hypothèse de l'origine sociale constitue la deuxième piste, avec trois interprétations possibles, non mutuellement exclusives. Selon la première, le mot « médiane » renverrait à la notion de médiation sociale, la société de tante Léonie étant un milieu d'interconnaissance où chacun se connaît et observe les comportements d'autrui. Dans cette perspective, l'interprétation suivante me paraît convaincante : la chambre de tante Léonie est au coeur

« d'un réseau très actif d'informations : Françoise, Eulalie, Théodore, le curé, tous les membres de la famille même font converger vers elle l'écho de ce qui se passe dans Combray. Elle remplace donc l'immédiateté de la possession oculaire par la médiation multiple du bavardage, du papotage, du cancan. Toute une thématique de l'ouï, de l'ouï-dire, fait d'elle un centre non plus seulement d'intégration, mais de renseignement, ou de classement, et même de prévision » (Richard $1974: 192)^{5}$.

Selon la deuxième interprétation, «médiane » pourrait désigner un milieu social et familial typé, celui de la province. D'une part, dans la Recherche, Proust souligne le caractère provincial de la chambre de tante Léonie. D'autre part, il était averti de la sociologie, une discipline naissante à son époque. Il n'y aurait donc rien d'étonnant à le voir associer le sens social de milieu, vulgarisé par le naturalisme dans les années 1880-1890, à la description d'une sensation olfactive.

Selon la troisième interprétation, la plus fragile, «médiane» aurait un sens médical (veine médiane), acception qui semble dominante au moins jusqu'à la mort de Proust et qui renvoie au milieu professionnel de son père, médecin et hygiéniste.

Ce serait donc triplement que « médiane » renverrait au milieu social, dans le sens de « réseau », de milieu sociologique et de milieu professionnel.

La troisième piste est liée à l'acception musicale du mot «médiane », sur laquelle je reviendrai plus loin. Pour l'instant, retenons simplement que ce mot, dans cette acception, a "mère » pour synonyme. Dans la Recherche, il est trivial de le rappeler, une grande partie du « temps retrouvé » est celui de l'enfance, cette longue période où le narrateur s'est «couché de bonne heure ». L'odeur médiane du couvre-lit serait alors une métonymie d'une tante certes paternelle mais néanmoins maternante ${ }^{6}$, elle-même évocatrice de la quête d'un retour in utero. Je n'irai pas plus loin sur cette piste proche de la psychanalyse, discipline dont je suis fort ignorant.

La quatrième piste semble a priori prometteuse puisqu'elle renvoie au langage des parfumeurs. On sait qu'ils distinguent les notes de tête (les composantes les plus volatiles et immédiatement perceptibles du parfum), les notes de cœur (les molécules les plus lourdes, qui donnent son caractère à la composition) et les notes de fond (l'arrière-plan durable). Or « médiane » peut être un synonyme de «note de cœur». Hélas, cet usage est tout à la fois extrêmement rare ${ }^{7}$, loin d'être consensuel chez les parfumeurs et, selon eux, absent des livres anciens de parfumerie. Il est donc improbable que Proust ait été inspiré par cette industrie.

La dernière piste littéraire est celle d'une influence du futurisme. Le 11 août 1913, le peintre Carlo Carrá, cofondateur de ce mouvement, signe un texte intitulé La pittura dei suoni, rumori, odori. Il y décrit « des sons, bruits et odeurs concaves ou convexes, triangulaires, ellipsoïdaux, oblongs, coniques, sphériques, spiraliques, etc. ${ }^{8}$. Son article est publié, à Florence, le 1er septembre 1913, dans le $\mathrm{n}^{\circ} 17$ de la revue Lacerba. Proust doit-il à ce texte l'idée d'une odeur « géométrique »? L'hypothèse n'est pas absurde, pour plusieurs raisons. En premier lieu, l'Italomanie de Proust est un fait établi. Ensuite, il était parfaitement informé des arts de son époque. Il n'a pas été ignorant du mouvement futuriste, explicitement mentionné dans $A$ l'ombre des jeunes filles en fleur (Proust 1987b : 449). Enfin, bête noire de ses éditeurs, Proust avait l'habitude d'intervenir très 
tardivement sur ses manuscrits. Bien que le travail de composition de Du côté de chez Swann se soit déroulé entre le 31 mars 1913 et le 14 mai 1913 (Yoshida 2005 : 32), on pourrait imaginer un ajout de dernière minute sur le manuscrit, achevé d'imprimer le 8 novembre et paru le 14 novembre 1913, soit plusieurs semaines après la publication de l'article de Carrá. Cette hypothèse, cependant, doit être écartée au vu des avant-textes. Proust écrivait et réécrivait, encore et encore, dans ses brouillons et manuscrits, de manière fragmentaire et dispersée. Or dans une page d'un cahier d'octobre $1909^{9}$ (ill. l) consacré à la description de la chambre de tante Léonie, on trouve mention de l'odeur " médiane ». Le premier usage de l'épithète est peut-être antérieur à ce cahier, mais cela suffit pour infirmer l'hypothèse d'une influence du texte de Carrá paru quatre ans plus tard. Doit-on pour autant exclure définitivement la possibilité que les idées futuristes aient pesé sur la prose proustienne ? Le Manifeste du mouvement est publié le 20 février 1909 dans Le Figaro, mais si le flair des fauves et «la bonne odeur de nos esprits pourrissants » y sont évoqués, on n'y trouve nulle trace d'odeurs géométriques. La question restera donc posée.

Ces cinq interprétations, empruntées aux études proustiennes ou suggérées par leur lecture, accroissent l'épaisseur sémantique du descripteur et, du même coup, offrent au lecteur une diversité des voies d'accès possibles à l'expérience olfactive du narrateur. D'autres pistes présentent l'avantage de mieux prendre en compte le contexte historique et, peut-être, un trait spécifique de la physiologie de Proust.

\section{Le contexte artistique et scientifique}

Les dictionnaires livrent plusieurs acceptions du mot «médian », en géométrie, en anatomie ${ }^{10}$, en architecture, en statistique, en linguistique et en musique, comme je l'ai déjà signalé. Selon Le Trésor de la langue française, la médiane est la note « qui se rencontre à la tierce (...) au-dessus de la finale [...]. Cette note médiane ou mère, qui divise chaque gamme jouait (...) un rôle dans la transposition. » Proust, en insérant dans son manuscrit cet étrange descripteur, a-t-il voulu signifier la proximité qu'il percevait entre le monde des sons et celui des odeurs?

Cette supposition n'est pas gratuite, si on considère le contexte artistique et scientifique de l'époque. Toute l'esthétique du XIXe et du début du XXe siècle a été influencée par l'apparition et le développement des théories de la perception, depuis le Traité des couleurs (1810) de Goethe jusqu'à l'Introduction à une esthétique scientifique (1885) de Charles Henry, pour ne citer que les contributions les plus connues. À travers la prise en compte de la psychologie des formes, de la physiologie des sensations ou de la théorie ondulatoire qui rapproche les modes de diffusion du son et de la lumière (Rousseau 2003 : 19-33), les artistes s'efforcent de saisir dans leurs œuvres le "grand monde de vibrations qui est à la base de l'univers » (Blanc-Gatti 1934: 24 cité in Roque 2003). Cette quête d'un langage universel, pansensoriel, celui des interconnexions et compénétrations sensorielles, anime les peintres au premier chef (Kandinsky, Kupka, Exter, Matiouchine et bien d'autres) qui conçoivent leurs toiles comme des symphonies chromatiques ou des fugues picturales, mais aussi les musiciens, (au projet de piano chromatique de la fin du XIXe siècle, on peut rattacher les recherches menées dès le XVIIIe siècle autour du clavecin oculaire) et, bien entendu, la littérature (e.g. les Correspondances, l'audition colorée des Voyelles, le symbolisme (Segalen 1981), l'esthétisme décadente huysmansienne). Dès lors, on ne prend pas grand risque en situant Proust, très attentif, on l'a dit, aux diverses expressions artistiques - mais aussi scientifiques (Lehrer 2007, Troscianko 2013) de son temps, dans ce courant esthétique oeuvrant pour une sorte de totalisation des divers mondes sensoriels et, peut-être, pour la découverte ou la conquête de leur harmonie universelle. Il me paraît significatif, de ce point de vue, que, vers 1892, dans le second " questionnaire de Proust ", l'auteur de la Recherche réponde ainsi à une question sur sa couleur préférée : «La beauté n'est pas dans les couleurs, mais dans leur harmonie. »"

Je pourrais arrêter ma glose imprudente du texte proustien sur ce constat d'une recherche d'un accord général des sens ou d'une résonance heureuse entre eux si une autre interprétation plausible, mais hélas invérifiable, ne m'avait été imposée par une intimité prolongée avec l'oeuvre. Loin d'être simplement marquée par une hyperesthésie frémissante, c'est une déferlante synesthésique qui la traverse tout entière, si bien qu'il paraît réducteur d'y voir seulement un banal " effet de littérature », celui d'un romancier reflétant fidèlement dans ses écrits la sensibilité artistique de son temps. J'introduis là mon hypothèse la plus aventureuse, celle d'un Proust synesthète. 


\section{La synesthésie de Proust}

Proust, supputè-je, avait des prédispositions physiologiques à la synesthésie, c'est-à-dire à une mise en correspondance involontaire des messages transmis par les différents sens. Le nombre de synesthètes dans une population est incertain, compte tenu de la diversité des formes de synesthésie (bimodale, multimodale, catégorielle ou cognitive) et de la difficulté qu'il y a à les observer. Selon les sources, l'estimation va de 4 à $5 \%$ des adultes à un sur 25.000 (Cytowic 1993, Simner et al. 2006). On pense, par ailleurs, bien que cela soit controversé, que la fréquence de la synesthésie est plus grande chez les poètes (Rimbaud, Baudelaire), les musiciens (Liszt, Messiaen), les peintres (la synesthésie bimodale de Kandinsky qui voyait ses couleurs en écoutant un opéra de Wagner) et les romanciers (la synesthésie catégorielle de Nabokov, pour qui la lettre $b$ évoquait le brun sienne et le $t$ le vert pistache $^{12}$, prédisposition qu'il tenait de sa mère et dont son fils, dit-on, a hérité). Sans être évidemment une preuve, ce dernier point donne un embryon de plausibilité à la synesthésie de Proust. Dans toute la Recherche, je l'ai déjà signalé, de nombreux exemples rendent vraisemblable cette thèse, soit parce que, à nouveau, Proust établit explicitement un lien direct entre l'ouïe et l'odorat (par exemple, quand il évoque "la fragrance de géranium» de la musique de Vinteuil (Proust 1987c : 299)) - soit parce qu'il ouvre plus largement la musique vers les autres sens. Je suis forcé de me limiter à quelques exemples, mais l'idéal proustien de pansensorialité semble sans bornes. Il associe goût, couleur et sonorité quand il évoque le « son doré des cloches » qui contient « la saveur fade des confitures » (Proust 1987c : 78); il réunit formes et couleurs avec l'ouie, « ce sens délicieux » qui « nous apporte la compagnie de la rue dont elle nous retrace toutes les lignes, dessine toutes les formes qui y passent, nous en montrant la couleur » (Proust 1987c : 103); il met en correspondance le son et la forme quand il décrit le "double tintement timide, ovale et doré de la clochette pour les étrangers " (Proust 1987a : 33) dans la maison familiale, à Combray. "Tout peut se transposer » (Proust 1987c : 79), affirme le narrateur, ce qui vient curieusement en écho des notes de Giacomo Balla, né la même année que Proust, dans un de ses carnets : «Tout s'abstrait par des équivalents qui de leur point de départ vont vers l'infini » (cité in Roque 2003 : 62). Pourquoi ne pas imaginer, alors, que l'auteur de la Recherche ait été enclin, par des prédispositions naturelles, à percevoir l'odeur du couvre-lit de tante Léonie en association à des sons (hypothèse musicale) ou à des espaces (hypothèse géométrique), ce qui l'aurait conduit à la qualifier de «médiane»?

Avec cette hypothèse hasardeuse, explorée par pur plaisir épistémique, je suis imprudemment sorti de mon domaine de compétence. Aussi vais-je essayer, dans la section conclusive, de renouer avec ma discipline en soutenant une hypothèse davantage anthropologique.

\section{Se méprendre (en partie) pour se comprendre}

La puissance communicative du langage tient notamment à sa capacité de rendre visible (dans le sens d'une présentification) ce qui est invisible, qu'il s'agisse d'une invisibilité contigente (par exemple, je peux parler d'un ami vivant aux antipodes) ou intrinsèque (par exemple, je peux parler de valeurs, d'un être surnaturel ou... d'une odeur). Les différentes déclinaisons de l' «ajoutage» proustien (Serça 2005) et les innombrables superpositions descriptives (Simon 1994) qui tissent la Recherche sont peut-être toutes au service d'une visibilité de l'invisible, c'est-à-dire de «l'effet que cela fait » pour le narrateur de goûter une madeleine trempée dans du thé, d'éprouver une joie liée à Venise au moment où les pavés mal équarris de la cour de l'hôtel des Guermantes lui rappellent " deux dalles inégales du baptistère de Saint-Marc » (Proust 1987d : 703), ou de se souvenir de l'odeur d'un couvre-lit. Cette expérience phénoménale étant réputée incommunicable (Nagel 1974), tout se passe comme si Proust s'efforçait de connoter par une saturation descriptive ce qu'il ne peut précisément dénoter. C'est bien ce que suggère Merleau-Ponty dans Le Visible et l'Invisible. «Personne n'a été plus loin que Proust, écrit-il, dans la fixation des rapports du visible et de l'invisible, dans la description d'une idée qui n'est pas le contraire du sensible, qui en est la doublure et la profondeur» (Merleau-Ponty 1996: 195). En accord avec l'hypothèse whorfienne (Candau 2003), la série descriptive dans laquelle s'inscrit l'épithète «médiane " permettrait au lecteur de se faire une idée de la singularité de la sensation et, pourquoi pas, d'en amorcer l'épreuve nonobstant sa fugacité. Seule l'association de plusieurs descripteurs serait à même de lui donner l'intuition des propriétés odorantes du couvre-lit et de la chambre, en provoquant des émotions qui leur seraient associées. 
Dans le rapprochement passionnant qu'il fait entre Lévi-Strauss, Platon et Proust, Marcel Hénaff note que chez ce dernier comme avec Mallarmé «s'affirme une pensée de l'écriture non plus comme geste démiurgique mais comme capacité d'entrer dans un espace de relations, d'activer des réseaux, d'en susciter de nouveaux ». De la même manière qu'une "variante en musique " traduit » un thème donné », c'est-à-dire le transforme et le développe » (Hénaff 2008 : 162), Proust joue et se joue des gammes olfactives, multipliant les épithètes pour, tel un impressionniste (Lacaille-Lefebvre 2011), s'approcher par touches et retouches successives de l'atmosphère olfactive qu'il cherche à peindre. Parce que « la perception primitive porte plutôt sur des relations que sur des termes isolés » (MerleauPonty 1964 : 27), c'est en fait une mosaïque olfactive que Proust élabore, chaque descripteur venant ponctuer et structurer l'identité odorante du couvre-lit de tante Léonie, elle-même étant un élément de la réalité que constitue pour le narrateur le paysage olfactif de la chambre.

«Ce que nous appelons la réalité, écrit Proust dans Le Temps retrouvé, est un certain rapport entre ces sensations et ces souvenirs qui nous entourent simultanément [...] rapport unique que l'écrivain doit retrouver pour en enchaîner à jamais dans sa phrase les deux termes différents " (Proust 1987d : 720).

Ce rapport associatif, opératoire et non-réflexif, note Hénaff, « est justement ce qui caractérise un processus symbolique » (Hénaff 2008: 168). Il n'est pas surprenant que Proust y excelle dans le registre des odeurs, celles-ci étant qualifiées par Sperber de « symboles par excellence »(Sperber 1974: 130), du fait de leur capacité à évoquer «des souvenirs et des sentiments soustraits à la communication sociale (...) en mettant directement en rapport la nature, et le for intérieur de celui qui l'observe ».

Cependant, ne peut-on craindre que l'apparente anarchie sémantique des descripteurs olfactifs de la chambre de tante Léonie (qu'y a-t-il de commun entre une odeur oisive, ou ponctuelle, ou médiane ?) nuise à ce partage sensoriel? Sans doute, s'il était certain qu'un langage fait de termes propres soit toujours la condition optimale de l'intercompréhension. Cette certitude, nous ne l'avons pas. Pourquoi existe-t-il des «jeux de langage» où règne l'ambivalence, voire l'imprécision qui, a priori, ne semblent pas favoriser le partage ? "Un langage fait de termes propres, observe saint Augustin dans Les Confessions, est chose rare : très souvent nous parlons sans propriété, mais on comprend ce que nous voulons dire. $\rangle^{13}$ Il est possible que la métaphore, très fréquente lors de la description d'une expérience olfactive, ne soit pas le fruit d'une perception mal lexicalisée (on ne trouverait pas «les mots pour le dire »), mais d'une exigence de la pensée humaine: garantir la réussite de la communication intersubjective. Or, cette réussite est très incertaine dès lors qu'il s'agit de communiquer des qualia, qualités subjectives définies comme l'expérience phénoménale de la chose (en l'occurrence, d'un stimulus olfactif). Si, publiquement, à l'aide de descripteurs rigides et transparents, nous cherchions à épuiser le contenu sémantique des représentations mentales associées à nos expériences olfactives - à supposer que nous en soyons capables -, ne prendrions-nous pas le risque de durcir à tel point les significations échangées que leur partage deviendrait impossible ? En revanche, nous parvenons à nous faire une certaine idée de ce que Proust veut exprimer par son usage du terme "médiane», la sédimentation sémantique et l'opacité ${ }^{14}$ relative de ce descripteur déconcertant suscitant de multiples interprétations, d'innombrables inférences qui, toutes réunies, nous rapprochent un peu, à petits pas, de l'expérience olfactive du narrateur. Certes, cette intercompréhension supposée est accompagnée d'une " mise entre guillemets » et même d'un certain degré de méprise puisque l'accord se fait sur une signification dont le partage reste hypothétique et, dans le meilleur des cas, partiel. Plusieurs auteurs (Dummett 1991: 146-147, Frege 1971: 105, Récanati 1979 : 78, Russell 1969 : 199, Sperber $1974: 111-113)$ ont abordé cette forme de cognition consistant en une suspension provisoire ou définitive de la dénotation habituelle d'une proposition au profit d'une compréhension plutôt évocatrice (une proposition «semi-propositionnelle»). Dans de nombreux cas, explique Russell, nous ne croyons pas que $p$ mais que « $p$ signifie une vérité ». Dans la vie quotidienne, cette mise entre guillemets a une certaine efficace. Lorsque le réparateur de mon ordinateur me dit qu'il y a un problème d'adresse sur sa carte-mère, je peux tenter d'excuser mon retard dans la livraison de mon dernier article en informant l'éditeur que je ne peux terminer mon texte parce que mon ordinateur " a un problème d'adresse ». J'ai de bonnes raisons de croire en la vérité de cet énoncé - ce réparateur est compétent, je sais vaguement que les informations sont retrouvées dans un ordinateur grâce à un système d'adresses - sans vraiment comprendre ce qu'il signifie exactement. Dans l'information que j'ai donnée à l'éditeur, j'ai dit que la carte-mère de mon ordinateur avait un 
problème d'adresse, mais on ne peut pas prétendre que je crois qu'une adresse ne fonctionne pas, on peut seulement affirmer que je crois que quelque chose qui s'appelle une adresse ne fonctionne pas. ${ }^{15}$

Ce qui est vrai de nos interactions sociales ordinaires l'est sans doute plus encore lors de la communication d'une expérience olfactive. La mise entre guillemets de nombreux descripteurs olfactifs, par exemple l'odeur médiane du couvre-lit de tante Léonie, est peut-être la condition nécessaire à une intercompréhension minimum entre Proust et ses lecteurs. Il faudrait alors admettre que dans le registre olfactif, nous nous comprenons parce que nous acceptons de nous méprendre en partie sur ce que nous comprenons. Autrement dit, l' 'impropriété » du langage naturel des odeurs serait le fruit et le signe de notre intelligence sociale et d'une quête du partage qui nous anime en permanence, tout autant qu'elle anima le narrateur.

\footnotetext{
${ }^{1}$ En 2004, j'ai tenté une première fois d'éclaircir cette énigme dans un article de la revue VOIR [barré] $\left(\mathrm{n}^{\circ} 28-\right.$ 29). Le présent texte reprend partiellement cette publication, en l'augmentant de nouvelles hypothèses. La version de 2004 devait beaucoup à mes collègues Béatrice Bloch (Université de Bordeaux 3) et Jean-Marc Quaranta (Université de Nice Sophia Antipolis). La version actuelle leur est tout autant redevable, ainsi qu'à Sophie Duval (université de Bordeaux 3) qui m'a permis d'écarter (provisoirement?) l'hypothèse futuriste, à Jean-Claude Ellena (parfumeur-compositeur chez Hermès), à Martine Sibre (doctorante à l'Université de Nice Sophia Antipolis) et à Marcel Vuillaume (professeur émérite à l’Université de Nice Sophia Antipolis).

${ }^{2}$ De même qu'il est improbable de trouver le mot rêche associé au toucher de la soie, il est contre-intuitif d'imaginer que l'épithète épais puisse qualifier le parfum de la violette.

${ }^{3} \mathrm{Si}$ on en juge par la rareté de ses descripteurs, la perception de l'odeur du couvre-lit par le narrateur est tout à fait singulière. Trois des épithètes sont des hapax dans la Recherche («médiane », "poisseuse », « fruitée »), « indigeste » n'y apparait que 2 fois, seul « fade » comptant 10 occurrences au singulier et 5 au pluriel (Brunet 1983).

${ }^{4}$ Cependant, il est dit un peu plus loin dans la Recherche que le lit « longeait la fenêtre »...

${ }_{6}^{5}$ Les italiques sont de moi.

${ }^{6}$ Pour Julia Kristeva (1994: 32), «tante Léonie suggère une version dérisoire de l'image maternelle que le narrateur n'aura aucun mal à désacraliser » J.-P. Richard (1974: 193) évoque l'intimité du lit de tante Léonie, « lieu médian » désirable visé par une pulsion inconsciente (la « convoitise inavouée »).

${ }^{7}$ Mais en anglais la note de coeur est la middle note, en espagnol la nota media...

${ }^{8}$ Dal punto di vista della forma: vi sono suoni, rumori e odori concavi e convessi, triangolari, elissoidali, oblunghi, conici, sferici, spiralici, ecc.

${ }^{9}$ Cahier 10 (NAF16650), folio 25 recto.

${ }^{10}$ Acception qui mériterait également d'être explorée, le père de Proust exerçant la profession de médecin.

${ }^{11}$ Questionnaire de Proust, «Biographie de Marcel Proust» in À la recherche du temps perdu. Du côté de chez Swann. À l'ombre des jeunes filles en fleurs, T. 1, Paris, Robert Laffont, 1987, p. 12.

${ }^{12}$ Sur ce type de perception, voir Robertson 2001.

${ }^{13}$ Saint Augustin, Les Confessions, XI, XX. Les italiques sont de moi.

${ }^{14}$ « Le signe est comme un miroir qui donne à voir autre chose que lui-même, ou bien encore il est comme une vitre transparente qui laisse voir autre chose qu'elle-même. Mais aussi bien le miroir que la vitre ont la propriété de s'opacifier, c'est-à-dire qu'ils peuvent cesser de se dérober pour au contraire s'offrir à la considération, à la vue de l'esprit. » (François 1979: 33).

${ }^{15} \mathrm{Je}$ transpose ici un dialogue imaginaire entre M. Dummett et son garagiste (1991 : 146-147).
}

\section{BIBLIOGRAPHIE}

Antoine Arnauld, Pierre Nicole, La logique ou l'art de penser, Paris, Gallimard, 1992.

Thierry Baccino, Daniel Cabrol-Bass, Joël Candau, Candice Meyer, Tobias Scheer, Marcel Vuillaume, Olivier Wathelet, "Sharing an Olfactory Experience: The Impact of Oral Communication", Food Quality and Preference, 21(5), 2010, 443-452.

Gaston Bachelard, La poétique de la rêverie, Paris, PUF, 1993.

Charles Blanc-Gatti, Des sons et des couleurs, Paris, Éditions d'art chromophonique, 1934.

Claude Boisson, "La dénomination des odeurs : variations et régularités linguistiques", Intellectica, 1(24), 1997, 29-49.

Étienne Brunet, Le vocabulaire de Proust, Genève-Paris, Slatkine, 1983.

Pierre Jean Georges Cabanis, 1980, Rapports du physique et du moral de l'homme, Genève, Slatkine Reprints, 1980.

Joël Candau, Mémoire et expériences olfactives, Paris, PUF, 2000.

Joël Candau, "De la ténacité des souvenirs olfactifs", La Recherche, 344, 2001, 58-62. 
Joël Candau, "El lenguaje natural de los olores y la hipótesis Sapir-Whorf", Revista de antropología social, 12, 2003, 243-259.

Joël Candau, "Les formes partagées des affects olfactifs : des protoaffects aux affects représentationnels", Noesis, 16, 2010a, 129-154.

Joël Candau, "Intersensorialité humaine et cognition sociale", Communications, 86, 2010b, 25-36.

Candau J., Jeanjean A.. 2006. « Des odeurs à ne pas regarder ». Terrain $47:$ 51-68.

Candau J., Wathelet O. 2011. « Les catégories d'odeurs en sont-elles vraiment? ». Langages 181: 7-52.

Cassirer Ernst, La philosophie des formes symboliques. 3. La phénoménologie de la connaissance, Paris, Minuit, 1972.

Chu S., Downes J. J. 2000. "Long live Proust : the odour-cued autobiographical memory bump". Cognition 75: B41-B50.

Richard E. Cytowic, The Man Who Tasted Shapes : A bizarre Medical Mystery Offers Revolutionary Insights into Reasoning, Emotion, and Consciousness, New York, Putnam, 1993.

Danièle Dubois, Catherine Rouby, "Une approche de l'olfaction : du linguistique au neuronal", Intellectica, 24, 1997/1, 9-20.

Michael Dummett, Les origines de la philosophie analytique, Paris, Gallimard, 1991.

Jean-Jacques Filippi, Xavier Fernandez, Elisabet Duñach, "Les composés volatils soufrés en chimie des arômes", Science des aliments, 27, 2007, 23-46.

Gottlob Frege, Écrits logiques et philosophiques, Paris, Seuil, 1971.

Marcel Hénaff, Claude Lévi-Strauss, le passeur de sens, Paris, Perrin, 2008.

Geneviève Idt, Littérature et langages (T. 3 : le roman, le récit non romanesque, le cinéma), Paris, Fernand Nathan, 1975.

Emmanuel Kant, Anthropologie d'un point de vue pragmatique, in Euvres philosophiques III, Paris, Gallimard, 1986.

Julia Kristeva, Le temps sensible. Proust et l'expérience littéraire, Paris, Gallimard, 1994.

Armelle Lacaille-Lefebvre, La poésie dans à la recherche du temps perdu de Marcel Proust, Paris, L'Harmattan, 2011.

Jonah Lehrer, Proust was a Neuroscientist, Boston - New York, Houghton Mifflin, 2007.

Claude Lévi-Strauss, Mythologiques. Le cru et le cuit, Paris, Plon, 1964.

Asifa Majid, Niclas Burenhult, "Odors are expressible in language, as long as you speak the right language", Cognition, 130(2), 2014, 266-270.

Maurice Merleau-Ponty, Le Visible et l'Invisible, Paris, Gallimard, 1964.

Maurice Merleau-Ponty, Le primat de la perception et ses conséquences philosophiques, Paris, Verdier, 1996.

Thomas Nagel, "What is Like to be a Bat". The Philosophical Review, 83(4), 1974, 433-450.

Steven Pinker, L'instinct du langage, Paris, Odile Jacob, 1999.

Marcel Proust, À la recherche du temps perdu. Du côté de chez Swann, Paris, Robert Laffont, 1987a.

Marcel Proust, À l'ombre des jeunes filles en fleurs, Paris, Robert Laffont, $1987 \mathrm{~b}$.

Marcel Proust, À la recherche du temps perdu. La Prisonnière, Paris, Robert Laffont, 1987c.

Marcel Proust, À la recherche du temps perdu. Le Temps retrouvé, Paris, Robert Laffont, 1987d.

François Récanati, La Transparence et l'Énonciation, Paris, Seuil, 1979.

Jean-Pierre Richard, Proust et le monde sensible, Paris, Seuil, 1974.

Lynn C. Robertson, "Colour my i's blue", Nature, 410, 2001, 533-534.

Georges Roque, "Ce grand monde des vibrations qui est à la base de l'univers" in Aux origines de l'abstraction 1800-1914, Paris, Éd. de la Réunion des musées nationaux, 2003, 51-67.

Pascal Rousseau, "Un langage universel. L'esthétique scientifique aux origines de l'abstraction" in Aux origines de l'abstraction 1800-1914, Paris, Éd. de la Réunion des musées nationaux, 2003, 19-33.

Bertrand Russell, Signification et vérité, Paris, Flammarion, 1969.

Saint Augustin, Les Confessions, Paris, GF-Flammarion, 1964.

Victor Segalen, Les synesthésies et l'école symboliste, Paris, Éditions Fata Morgana, 1981.

Isabelle Serça, "Les différentes déclinaisons de 1'« ajoutage » proustien", Bulletin d'informations proustiennes $35,2005,67-76$.

Julia Simner, Catherine Mulvenna, Noam Sagiv, Elias Tsakanikos, Sarah A. Witherby, Christine Fraser, Kirsten Scott, Jamie Ward, "Synaesthesia: the prevalence of atypical cross-modal experiences", Perception 35, 2006, 1024-1033.

Anne Simon, "Proust et la superposition descriptive", Bulletin d'informations proustiennes 25, 1994, 151-166.

Dan Sperber, Le symbolisme en général, Paris, Hermann, 1974.

Emily T. Troscianko, "Cognitive realism and memory in Proust's madeleine episode", Memory Studies, 6(4), 2013, 437-456.

Georges Vigarello, Le propre et le sale. L'hygiène du corps depuis le Moyen Age, Paris, Seuil, 1985. 
Jo Yoshida, "Ce que nous apprennent les épreuves de Du côté de chez Swann dans la collection Bodmer", Bulletin d'informations proustiennes, 35, 2005, 31-45. 